\title{
B7-H4 overexpression correlates with a poor prognosis for cervical cancer patients
}

\author{
WENTING LIU ${ }^{1}$, KIYOSUMI SHIBATA ${ }^{2}$, YOSHIHIRO KOYA ${ }^{3}$, HIROAKI KAJIYAMA ${ }^{2}$, \\ TAKESHI SENGA ${ }^{4}$, MAMORU YAMASHITA ${ }^{1}$ and FUMITAKA KIKKAWA ${ }^{2}$ \\ ${ }^{1}$ Bell Research Center for Reproductive Health and Cancer, Kishokai Medical Corporation, Nagoya, Aichi 458-0801; \\ ${ }^{2}$ Department of Obstetrics and Gynecology, Nagoya University Graduate School of Medicine, Nagoya, Aichi 466-8550; \\ ${ }^{3}$ Bio-Databases Institute of Reproductive and Developmental Medicine, Nagoya, Aichi 458-0801; \\ ${ }^{4}$ Division of Cancer Biology, Nagoya University Graduate School of Medicine, Nagoya, Aichi 466-8550, Japan
}

Received October 16, 2013; Accepted December 5, 2013

DOI: $10.3892 / \mathrm{mco} .2013 .225$

\begin{abstract}
Cervical cancer is a major global public health care concern and the second most commonly diagnosed malignancy among females worldwide. B7-H4 is an immunoregulatory protein that has been shown to be overexpressed in several types of cancer and is often associated with more advanced disease and poor prognosis. We investigated whether B7-H4 is a prognostic maker for cervical cancer by detecting its expression in cervical cancer specimens. Formalin-fixed, paraffin-embedded tissue blocks from cervical cancer were evaluated for B7-H4 expression by immunohistochemistry with free R software analysis. The intensity of B7-H4 immunoexpression was evaluated according to age, histological type, International Federation of Gynecology and Obstetrics (FIGO) stage, lymphovascular space invasion (LVSI) and lymph node status. We investigated the distribution and expression of B7-H4 in 102 cervical cancer specimens and determined the association between its expression and clinicopathological characteristics, including patient outcomes. Of the 102 specimens, 31 were found to be negative for B7-H4 immunoexpression, whereas 71 were B7-H4-positive. When classified by negative vs. positive expression, B7-H4 was not found to be associated with any of the clinicopathological parameters investigated. A positive B7-H4 expression significantly predicted poor overall survival $(\mathrm{OS})$ when compared to negative expression $(\mathrm{P}<0.05)$. In the multivariate analysis, positive $\mathrm{B} 7-\mathrm{H} 4$ expression was identified as an independent prognostic factor for OS $(\mathrm{P}<0.05)$. Our data suggested that positive B7-H4 expression may be a useful biomarker in patients with cervical cancer likely to have an unfavorable clinical outcome.
\end{abstract}

Correspondence to: Dr Kiyosumi Shibata, Department of Obstetrics and Gynecology, Nagoya University Graduate School of Medicine, 65 Tsurumai-cho, Showa-ku, Nagoya, Aichi 466-8550, Japan

E-mail: shiba@med.nagoya-u.ac.jp

Key words: B7-H4, cervical cancer, progression, immunoregulation

\section{Introduction}

Cervical cancer develops from the cells that constitute the cervical epithelium and involves an abnormal increase in the number of immature cells in this region. Cervical cancer is the second most commonly diagnosed malignancy among females worldwide according to the International Federation of Gynecology and Obstetrics (FIGO) (1). Despite the generally favorable prognosis of early cervical cancer patients, approximately one-third of these patients eventually succumb to the disease.

The optimal treatment selection for cervical cancer is multidisciplinary and is based on several factors, including patient age, histological subtype, tumor grade, stage and lymph node status, depth of stromal invasion and lymphovascular space invasion (LVSI). The immunoresponse was also identified as an important prognostic factor for survival in cervical cancer (2). B7 family members and their receptors are crucial in the regulation of antigen-specific immune responses (3).

The modulation and suppression of antitumor immune response is a characteristic that enables tumor cells to escape immune surveillance. Members of the B7 family are involved in this process, since the level of activation of the antitumor immune response depends on the balance between co-stimulatory and co-inhibitory signals. Certain molecules, which are often overexpressed in tumors, have been associated with the pathogenesis and progression of malignancies, as well as their immunological and non-immunological functions. The B7 homologs play a key role in the maintenance of self-tolerance and regulation of innate and adaptive immunity in tumor-bearing hosts (4).

B7-H4 (also known as B7x and B7S1) is the most recently identified immunoregulatory member of the B7 family $(3,5,6)$. B7-H4 has been implicated in the inhibition of T-cell-mediated immunity and the downregulation of the $\mathrm{T}$-cell response through the inhibition of T-cell proliferation, cytokine production and cell cycle progression $(3,7,8)$. Although the expression of B7-H4 is characteristic in lymphoid cells, B7-H4 mRNA is highly expressed in human breast and ovarian cancers, but not in the majority of normal tissues (9-11). Aberrant B7-H4 expression was also demonstrated in the cancer cells of patients with various malignancies, such as melanoma, ovarian, gastric 
and non-small-cell lung cancer, renal cell carcinoma, esophageal and endometrial cancer, and its expression was found to be correlated with the progression of the disease (7,8,11-18). Cervical cancer is difficult to detect early and it may also occur at a young age. While early detection improves the cure rate, the stage at diagnosis remains one of the most significant prognostic factors. Therefore, it is crucial to identify novel biomarkers for early detection and new targets for the treatment of cervical cancer. B7-H4 is reportedly overexpressed in early-stage ovarian cancer and is independent of CA125, suggesting that B7-H4 may be a novel biomarker (19).

B7-H4 was shown to be a prognostic maker in various tumors, although the correlation between B7-H4 expression and the prognosis of cervical cancer has not been fully elucidated. In this study, we measured the B7-H4 expression intensity in cervical cancer tissues using an immunohistochemical method and the association of B7-H4 expression with clinicopathological parameters and the intensity of expression was analyzed. B7-H4 may serve as a novel prognostic predictor for human cervical cancer and is also a potential target for therapeutic intervention.

\section{Materials and methods}

Tissue samples. Tissues were obtained from 102 patients who underwent surgery at the Nagoya University Hospital. The age of the patients ranged between 23 and 80 years, with a median age of 50 years. Patients who underwent pre-operative treatment, such as radiotherapy and/or chemotherapy, were excluded. All tissue samples were fixed in $10 \%$ formalin, embedded in paraffin and routinely stained with hematoxylin and eosin for histological examination.

Immunohistochemical B7-H4 staining and evaluation. Formalin-fixed, paraffin-embedded tissue blocks were cut into 4-mm sections and mounted on charged glass slides, deparaffinized and rehydrated in a graded series of ethanol. Antigen retrieval was performed in $1 \mathrm{mmol} / \mathrm{l}$ EDTA solution (Nacalai Tesque Inc., Kyoto, Japan) (pH 8.0) at $98^{\circ} \mathrm{C}$ for $15 \mathrm{~min}$. Endogenous peroxidase activity was blocked with $3.0 \%$ hydrogen peroxide (Wako, Osaka, Japan) for $5 \mathrm{~min}$ at room temperature. Blocking was performed in $10 \%$ normal goat serum (NGS; DakoCytomation, Glostrup, Denmark) with $1 \%$ bovine serum albumin (Sigma-Aldrich, St. Louis, MO, USA) in Tris-buffered saline (TBS) with $0.025 \%$ Tween-20 (TBS-T, Kanto Chemical Co., Inc., Tokyo, Japan) for $60 \mathrm{~min}$. The sections were incubated with anti-B7-H4 rabbit monoclonal antibody in blocking buffer at $4^{\circ} \mathrm{C}$ overnight (clone EP1165; Abcam, Cambridge, MA, USA). After three washings, the sections were incubated with biotinylated goat anti-rabbit horseradish peroxidase for $60 \mathrm{~min}$ at a 1:500 dilution, followed by incubation with avidin-biotin complex reagent for $30 \mathrm{~min}$ at a 1:100 dilution (PK-4001; Vector Laboratories, Burlingame, CA, USA) and finally visualized with 3,3'-diaminobenzidine (DAB peroxidase substrate kit, SK-4100, Vector Laboratories) in Milli-Q purified water (Millipore, Billerica, MA, USA). The slides were counterstained with Mayer's hematoxylin (Wako). The sections were dehydrated, cleared and mounted. Negative controls were run on all sections with $3 \%$ NGS in TBS-T, generated against unrelated antigens. The intensity
Table I. Association between B7-H4 expression and clinicopathological parameters.

\begin{tabular}{|c|c|c|c|c|}
\hline \multirow[b]{2}{*}{ Parameters } & \multirow[b]{2}{*}{$\mathrm{n}$} & \multicolumn{2}{|c|}{ B7-H4 } & \multirow[b]{2}{*}{ P-value } \\
\hline & & $\begin{array}{c}\text { Negative } \\
(\%)\end{array}$ & $\begin{array}{l}\text { Positive } \\
(\%)\end{array}$ & \\
\hline Total & 102 & $31(30.4)$ & 71 (69.6) & - \\
\hline \multicolumn{5}{|l|}{ Age (years) } \\
\hline$<50$ & 53 & $15(28.3)$ & 38 (71.7) & \multirow[t]{2}{*}{0.793} \\
\hline$\geq 51$ & 49 & $16(32.7)$ & $33(67.3)$ & \\
\hline \multicolumn{5}{|l|}{ FIGO stage } \\
\hline I-IIA & 73 & 24 (32.9) & 49 (67.1) & \multirow[t]{2}{*}{0.531} \\
\hline IIB-IV & 29 & $7(24.1)$ & $22(75.9)$ & \\
\hline \multicolumn{5}{|l|}{ Histological type } \\
\hline Squamous & 83 & $27(32.5)$ & $56(67.5)$ & \multirow[t]{2}{*}{0.481} \\
\hline Adenocarcinoma & 19 & $4(21.1)$ & $15(78.9)$ & \\
\hline \multicolumn{5}{|l|}{ LVSI } \\
\hline Negative & 54 & $14(25.9)$ & $40(74.1)$ & \multirow[t]{2}{*}{0.410} \\
\hline Positive & 48 & $17(35.4)$ & 31 (64.6) & \\
\hline \multicolumn{5}{|l|}{ Lymph node } \\
\hline Negative & 74 & $22(29.7)$ & $52(70.3)$ & \multirow[t]{2}{*}{0.996} \\
\hline Positive & 28 & $9(32.1)$ & $19(67.9)$ & \\
\hline
\end{tabular}

FIGO, International Federation of Gynecology and Obstetrics; LVSI, lymphovascular space invasion.

of immunostaining for B7-H4 was scored as 0 (negative), 1 (weak), 2 (medium) and 3 (strong).

Statistical analysis. The association between negative vs. positive B7-H4 expression and the clinicopathological parameters was evaluated using $\chi^{2}$ tests. The various clinicopathological parameters were evaluated using the Kaplan-Meier method by univariate survival analysis. The comparison between the survival curves was analyzed using the log-rank test for differences in overall patient survival. The overall survival (OS) was defined as the time between the date of surgery and last date of follow-up or date of death from cervical cancer. The disease-free survival (DFS) was defined as the time interval between the date of surgery and date of recurrence or that of the last follow-up. The prognostic significance of B7-H4 expression according to other pathological variables was assessed using the multivariate Cox's proportional hazards analysis. Statistical analyses were conducted using free $\mathrm{R}$ software and $\mathrm{P}<0.05$ was considered to indicate a statistically significant difference.

\section{Results}

Immunohistochemical detection of B7-H4 in cervical cancer tissues. A total of 102 cervical cancer specimens were investigated in this study and $71(69.6 \%)$ were found to be positive for B7-H4 immunoexpression, of which 23 were strongly positive. B7-H4 was detected on the cell membrane as well as in the cytoplasm of cervical cancer cells (Fig. 1). In several cases, the 

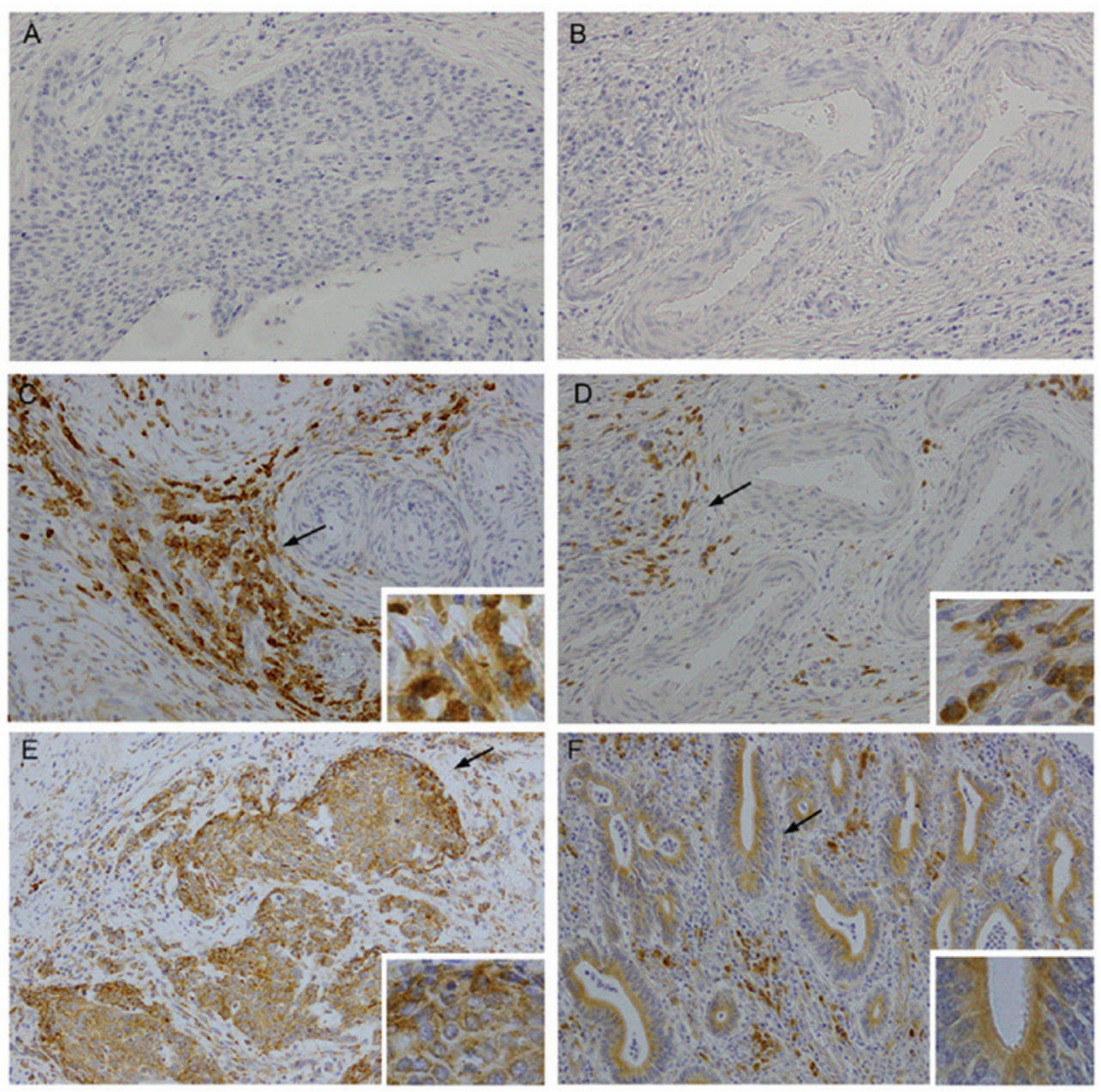

Figure 1. Immunohistochemical analysis of B7-H4 in cervical cancer. (A and B) Representative results of negative control expression in squamous cell carcinoma and vascular spaces; (C and D) the cancer cells involving vascular spaces were strongly stained in certain cases. Positive B7-H4 expression in (E) squamous cell carcinoma and (F) adenocarcinoma. Arrows, B7-H4 positivity expressed in the cytoplasm and on the membrane of cervical cancer cells; boxes, magnification of the respective areas indicated by the arrows.

expression of $\mathrm{B} 7-\mathrm{H} 4$ in the tumor stroma was found to be higher compared to that in tumor cells (Fig. 1C and D). There were also cases in which cancer involving vascular spaces exhibited strong B7-H4 staining (Fig. 1C and D). The strongly positive cases were included in positive cases for the statistical analysis. The association between B7-H4 expression and clinicopathological variables is shown in Table I. There was no significant correlation between the expression of B7-H4 and patient age, FIGO stage, histological type, LVSI, or lymph node status. Representative images of B7-H4 staining are shown in Fig. 1.

Association of B7-H4 expression with the survival of cervical cancer patients. The median follow-up for all patients was 68 months (range, 4.8-169 months). Of the 102 patients, $20(19.6 \%)$ had developed a relapse of cervical cancer at the time of the last follow-up and 16 (15.6\%) eventually succumbed to the disease. The 5-year OS rate for patients who were negative $(n=31)$ and positive $(n=71)$ for B7-H4 expression were 94.7 and $78.4 \%$, respectively (Table II). Fig. 2 shows the OS and DFS curves with respect to B7-H4 expression. The OS of B7-H4-positive patients was significantly lower compared with that of B7-H4-negative patients $(\mathrm{P}=0.014)$. The DFS in B7-H4-positive patients exhibited a tendency to be associated with positive B7-H4 expression, although this association was not significant $(\mathrm{P}=0.0813)$.

In the univariate analysis, age, FIGO stage, positive LVSI and lymph node metastasis and positive expression of B7-H4 were found to be significant predictors of poor OS. Furthermore, FIGO stage and lymph node metastasis, which are prognostic factors of DFS, were significantly poorer in B7-H4-positive cases. Other factors, including age and LVSI, exhibited a tendency to be associated with DFS, although this association was not significant (Table II). Notably, with regard to OS and DFS, FIGO stage and positive lymph node metastasis were found to be more significant $(\mathrm{P}<0.01$ and $<0.001$, respectively).

The cases were subdivided further into two groups according to their FIGO stage (early stage I-IIA and advanced stage IIB-IV). In the early-stage group, B7-H4 expression was significantly associated with prognosis $(\mathrm{P}=0.0372)$ (Fig. 3A). However, in the advanced-stage group, B7-H4 expression was not significantly associated with prognosis $(\mathrm{P}=0.207)$ (Fig. 3B).

Similarly, all the cases were stratified according to histological subtype into squamous cell carcinoma (SCC) and adenocarcinoma (Fig. 3C and D). The expression of B7-H4 was a significant prognostic factor in SCC, but not in adenocarcinoma 
Table II. Univariate analysis of various clinicopathological parameters in relation to survival of patiens with cervical cancer.

\begin{tabular}{|c|c|c|c|c|c|}
\hline \multirow[b]{2}{*}{ Parameters } & \multirow[b]{2}{*}{$\mathrm{n}$} & \multicolumn{2}{|c|}{ Overall survival } & \multicolumn{2}{|c|}{ Disease-free survival } \\
\hline & & 5-year survival (\%) & P-value & 5-year survival (\%) & P-value \\
\hline \multicolumn{6}{|l|}{ Age (years) } \\
\hline$<50$ & 53 & 90.6 & 0.0386 & 88.4 & 0.0685 \\
\hline$\geq 51$ & 49 & 79.0 & & 74.6 & \\
\hline \multicolumn{6}{|l|}{ FIGO stage } \\
\hline I-IIA & 73 & 90.3 & 0.0077 & 90.3 & 0.0001 \\
\hline IIB-IV & 29 & 71.5 & & 59.1 & \\
\hline \multicolumn{6}{|l|}{ Histological type } \\
\hline Squarmous & 83 & 85.4 & 0.6410 & 82.6 & 0.4700 \\
\hline Adenocarcinoma & 19 & 83.0 & & 78.3 & \\
\hline \multicolumn{6}{|l|}{ LVSI } \\
\hline Negative & 54 & 90.6 & 0.0387 & 88.7 & 0.0679 \\
\hline Positive & 48 & 79.1 & & 74.2 & \\
\hline \multicolumn{6}{|l|}{ Lymph node status } \\
\hline Negative & 74 & 90.4 & 0.0009 & 88.9 & 0.0009 \\
\hline Positive & 28 & 70.4 & & 62.9 & \\
\hline \multicolumn{6}{|l|}{ B7-H4 } \\
\hline Negative & 31 & 94.7 & 0.0140 & 90.2 & 0.0813 \\
\hline Positive & 71 & 78.4 & & 78.2 & \\
\hline
\end{tabular}

FIGO, International Federation of Gynecology and Obstetrics; LVSI, lymphovascular space invasion.

Table III. Multivariate analysis of several clinicopathological parameters in relation to survival of patiens with cervical cancer.

\begin{tabular}{|c|c|c|c|c|}
\hline \multirow[b]{2}{*}{ Parameters } & \multicolumn{2}{|c|}{ Overall survival } & \multicolumn{2}{|c|}{ Disease-free survival } \\
\hline & Relative risk $(95 \% \mathrm{CI})$ & P-value & Relative risk $(95 \% \mathrm{CI})$ & P-value \\
\hline \multicolumn{5}{|l|}{ Age (years) } \\
\hline$<50$ & 1 & 0.098 & 1 & 0.220 \\
\hline$\geq 51$ & $2.54(0.841-7.659)$ & & $1.82(0.694-4.783)$ & \\
\hline \multicolumn{5}{|l|}{ FIGO stage } \\
\hline I-IIA & 1 & 0.240 & 1 & 0.016 \\
\hline IIB-IV & $1.86(0.658-5.268)$ & & $3.47(1.263-9.551)$ & \\
\hline \multicolumn{5}{|l|}{ Histological type } \\
\hline Squarmous & 1 & 0.800 & 1 & 0.470 \\
\hline Adenocarcinoma & $0.86(0.261-2.805)$ & & $1.49(0.503-4.434)$ & \\
\hline \multicolumn{5}{|l|}{ LVSI } \\
\hline Negative & 1 & 0.220 & 1 & 0.230 \\
\hline Positive & $2.27(0.615-8.405)$ & & $2.02(0.640-6.360)$ & \\
\hline \multicolumn{5}{|l|}{ Lymph node status } \\
\hline Negative & 1 & 0.200 & 1 & 0.300 \\
\hline Positive & $2.32(0.642-8.349)$ & & $1.83(0.578-5.787)$ & \\
\hline \multicolumn{5}{|l|}{ B7-H4 } \\
\hline Negative & 1 & 0.025 & 1 & 0.072 \\
\hline Positive & $10.34(1.345-79.478)$ & & $3.19(0.903-11.247)$ & \\
\hline
\end{tabular}

CI, confidence interval; FIGO, International Federation of Gynecology and Obstetrics; LVSI, lymphovascular space invasion. 

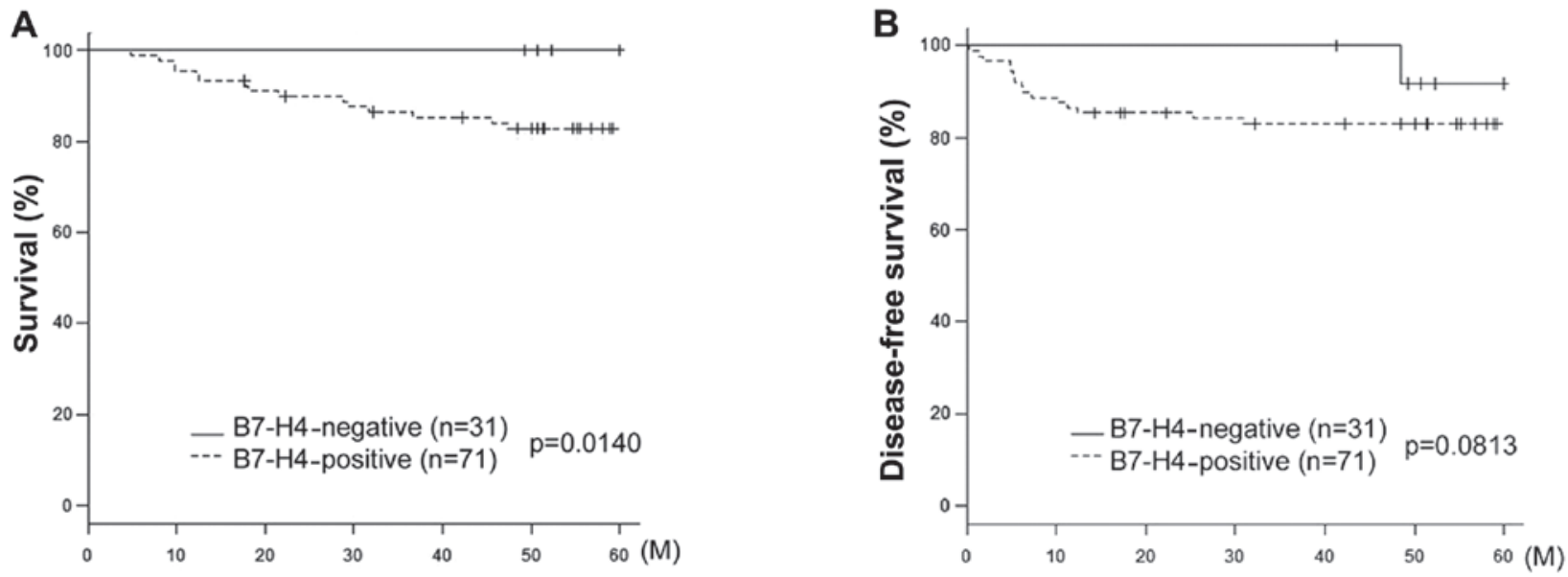

Figure 2. Kaplan-Meier survival curves for primary cervical cancer according to the immunoexpression of B7-H4. (A) Overall survival (OS). (B) Disease-free survival (DFS). Continuous line, negative B7-H4 expression ( $\mathrm{n}=31)$. Discontinuous line, positive B7-H4 expression ( $\mathrm{n}=71)$. The patients with tumors exhibitingB7-H4 expression had a significantly poorer carcinoma-specific survival (OS, $\mathrm{P}=0.0140$; $\mathrm{DFS}, \mathrm{P}=0.0813)$.
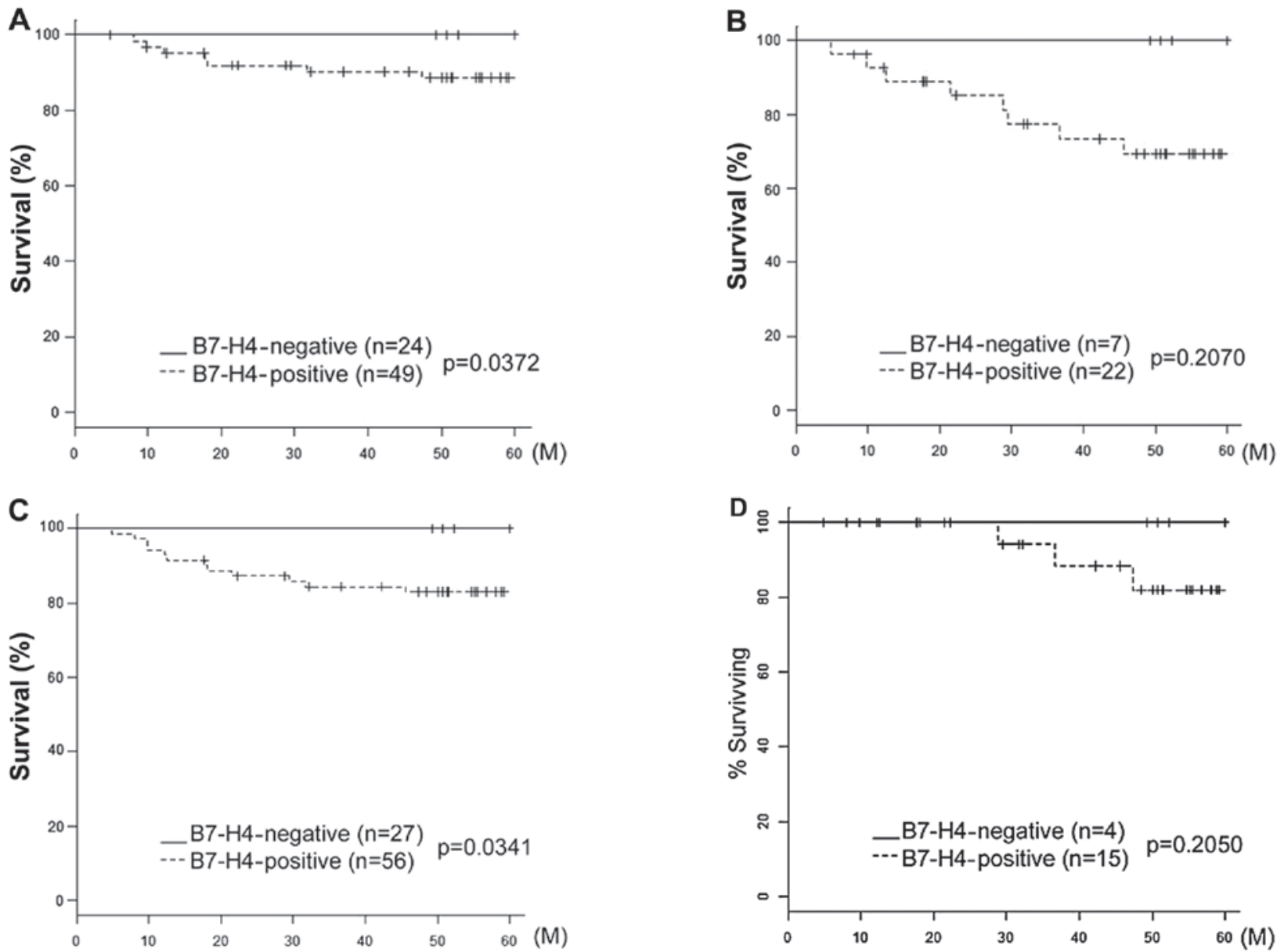

Figure 3. Carcinoma-specific overall survival rate of cervical cancer patients with tumors exhibiting B7-H4 expression. Kaplan-Meier survival curves for primary cervical cancer according to the immunoexpression of B7-H4 stratified by International Federation of Gynecology and Obstetrics (FIGO) stage and histological type. (A and B) Kaplan-Meier survival curves stratified by FIGO stage (A, stages I-IIA and B, stages IIB-IV). (C and D) Kaplan-Meier survival curves stratified by histological type (C, squamous cell carcinoma and D, adenocarcinoma). Continuous line, negative B7-H4 expression. Discontinuous line, positive B7-H4 expression. Stage I-IIA, IIB-IV and squamous cell carcinoma exhibiting B7-H4 expression had a significantly poorer carcinoma-specific survival $(\mathrm{P}=0.0372,0.207$ and 0.0341 , respectively).

( $\mathrm{SCC}, \mathrm{P}=0.0341$ and adenocarcinoma, $\mathrm{P}=0.205$ ). However, the squamous cell carcinoma subtype was a significant poor prognostic factor.
Multivariate analysis of prognostic variables in cervical cancer patients. As shown in Table III, in the multivariate OS analysis, age, FIGO stage, histological type, presence of LVSI, 
lymph node status and B7-H4 expression were included in the Cox proportional hazards analysis. The relative risk (RR) for positive B7-H4 expression was 10.34 and the $95 \%$ confidence interval (CI) was 1.345-79.478; $\mathrm{P}=0.025$. Similarly, the multivariate analysis of DFS revealed that only the FIGO stage was a significant independent prognostic factor $(\mathrm{R}=3.47$; 95\% CI: 1.263-9.551; $\mathrm{P}=0.016)$. However, age, histological type, LVSI, and lymph node status were not significantly associated. With regard to DFS, there was a difference between positive and negative expression of B7-H4, but it was not considered to be a significant independent prognostic factor, due to the narrow margin ( $\mathrm{RR}=3.19$; 95\% $\mathrm{CI}$ : 0.903-11.247; $\mathrm{P}=0.072)$.

\section{Discussion}

In the present study of 102 cervical cancer patients with a long-term follow-up, our results demonstrated that B7-H4 was highly and aberrantly expressed $(71 / 102,69.6 \%)$ and the B7-H4 expression level was not associated with any of the clinicopathological parameters. B7-H4 immunoexpression was evaluated based on stain intensity and area analysis. However, there was no significant association between B7-H4 expression and OS excluding intensity analysis. High B7-H4 expression was observed in a predominantly cytoplasmic and circumferential membranous distribution pattern. There was also limited immmunoreactivity for B7-H4 in the tumor stroma and perivascular area. Similar results were previously reported (20). In addition to tumor cells, tumor-infiltrating macrophages $(8,15,21)$ and the endothelial cells of small blood vessels in the cancer microenvironment were also found to constitutively express B7-H4. B7-H4 was also found to be highly expressed on tumor-associated macrophages in the ascites of ovarian cancer patients and may contribute to tumor progression (15).

In our study, high tumor B7-H4 expression was associated with a poorer OS in the uni- and multivariate analysis of all the factors affecting survival. The correlation between B7-H4 expression and its clinical significance in patients with malignancies was previously described $(7,10,11,15-17,20,22,23)$. Despite the unique patient populations, the differences in the antibodies used, the variations in the definitions of endpoints and the different pathologists evaluating the specimens, we independently obtained markedly similar results. Those studies suggested that aberrantly expressed B7-H4 was associated with adverse pathological characteristics and an increased risk of a poor prognosis or death from this disease. Our results demonstrated that cervical cancer patients with B7-H4-positive tumors exhibited a significantly worse OS compared to that of B7-H4-negative patients.

B7-H4 staining intensity was found to be associated with cancer spread along with clinical cancer recurrence and subsequent death. The invasion of lymphatic or blood vessels by carcinomatous cells is considered to be a critical step in the formation of lymphatic or distant metastasis. The involvement of lymphatic vessels is clearly an indicator of an unfavorable prognosis, even in early-stage cervical cancer. Tumor B7-H4 expression was also associated with lymphocytic infiltration, which predicts a poor clinical outcome for patients with cervical cancer (7). Furthermore, we observed that B7-H4 was associated with a significantly increased mortality risk in cervical cancer patients with pathologically localized cervical cancer tumors in the univariate analysis. Although FIGO stage, LVSI and lymph node metastasis were not identified as significant independent prognostic factors, they tended to be associated with poor prognosis. B7-H4 expression in cervical cancer patients is an independent prognostic predictor of the clinical outcome.

The role of B7-H4 has not been clearly determined and functional analyses are currently difficult to perform. B7-H4 was demonstrated to be a negative regulator of T-cell responses and to render tumors cells refractory to apoptosis $(9,13)$. Regarding the underlying mechanism, one possible explanation is that B7-H4 expression inhibits T-cell proliferation, decreases cytokine secretion and prevents apoptosis $(8,13)$. Two studies demonstrated that the overexpression of B7-H4 in human ovarian cancer cell lines promoted tumor formation in severe combined immunodeficiency mice, whereas short interfering RNA-mediated knockdown of B7-H4 mRNA and protein expression in a breast cancer cell line enhanced intracellular caspase activity, leading to the acceleration of tumor cell apoptosis (9,24). Furthermore, B7-H4 was shown to affect the immune response to and the outcome of lethal pulmonary infection (25) and induce donor-specific tolerance following transplantation $(26,27)$. Therefore, B7-H4 may also be used as an immunotherapeutic target against malignancies.

In conclusion, in this study, we presented evidence supporting the role of $\mathrm{B} 7-\mathrm{H} 4$ as an independent prognostic predictor of clinical outcome in cervical cancer patients. Further functional experiments may elucidate the molecular mechanism underlying the effect of B7-H4 on the prognosis of cervical cancer. Furthermore, B7-H4 may be a novel target for molecular-targeted therapy against cervical cancer.

\section{Acknowledgements}

The authors would like to thank Dr Luo and Ms. Daimon for their laboratory support.

\section{References}

1. Parkin DM, Bray F, Ferlay $\mathrm{J}$ and Pisani P: Estimating the world cancer burden: Globocan 2000. Int J Cancer 94: 153-156, 2001.

2. Shibata K, Kajiyama H, Ino K, et al: Twist expression in patients with cervical cancer is associated with poor disease outcome. Ann Oncol 19: 81-85, 2008.

3. Sica GL, Choi IH, Zhu G, et al: B7-H4, a molecule of the B7 family, negatively regulates $\mathrm{T}$ cell immunity. Immunity 18 : 849-861, 2003

4. Fauci JM, Straughn JM Jr, Ferrone S and Buchsbaum DJ: A review of B7-H3 and B7-H4 immune molecules and their role in ovarian cancer. Gynecol Oncol 127: 420-425, 2012.

5. Prasad DV, Richards S, Mai XM and Dong C: B7S1, a novel B7 family member that negatively regulates $\mathrm{T}$ cell activation. Immunity 18: 863-873, 2003.

6. Zang X, Loke P, Kim J, Murphy K, Waitz R and Allison JP: B7x: a widely expressed B7 family member that inhibits T cell activation. Proc Natl Acad Sci USA 100: 10388-10392, 2003.

7. Krambeck AE, Thompson RH, Dong H, et al: B7-H4 expression in renal cell carcinoma and tumor vasculature: associations with cancer progression and survival. Proc Natl Acad Sci USA 103: 10391-10396, 2006.

8. Kryczek I, Wei S, Zhu G, et al: Relationship between B7-H4, regulatory $\mathrm{T}$ cells, and patient outcome in human ovarian carcinoma. Cancer Res 67: 8900-8905, 2007.

9. Salceda S, Tang T, Kmet M, et al: The immunomodulatory protein $\mathrm{B} 7-\mathrm{H} 4$ is overexpressed in breast and ovarian cancers and promotes epithelial cell transformation. Exp Cell Res 306: $128-141,2005$. 
10. Tringler B, Zhuo S, Pilkington G, et al: B7-H4 is highly expressed in ductal and lobular breast cancer. Clin Cancer Res 11: 1842-1848, 2005.

11. Tringler B, Liu W, Corral L, et al: B7-H4 overexpression in ovarian tumors. Gynecol Oncol 100: 44-52, 2006.

12. Chen LJ, Sun J, Wu HY, et al: B7-H4 expression associates with cancer progression and predicts patient's survival in human esophageal squamous cell carcinoma. Cancer Immunol Immunother 60: 1047-1055, 2011.

13. Seliger B, Marincola FM, Ferrone S and Abken H: The complex role of B7 molecules in tumor immunology. Trends Mol Med 14: 550-559, 2008.

14. Quandt D, Fiedler E, Boettcher D, Marsch WCh and Seliger B: B7-H4 expression in human melanoma: its association with patients' survival and antitumor immune response. Clin Cancer Res 17: 3100-3111, 2011.

15. Kryczek I, Zou L, Rodriguez P, et al: B7-H4 expression identifies a novel suppressive macrophage population in human ovarian carcinoma. J Exp Med 203: 871-881, 2006.

16. Miyatake T, Tringler B, Liu W, et al: B7-H4 (DD-O110) is overexpressed in high risk uterine endometrioid adenocarcinomas and inversely correlated with tumor T-cell infiltration. Gynecol Oncol 106: 119-127, 2007.

17. Sun Y, Wang Y, Zhao J, et al: B7-H3 and B7-H4 expression in non-small-cell lung cancer. Lung Cancer 53: 143-151, 2006.

18. Arigami T, Uenosono $\mathrm{Y}$, Ishigami S, Hagihara T, Haraguchi $\mathrm{N}$ and Natsugoe S: Clinical significance of the B7-H4 coregulatory molecule as a novel prognostic marker in gastric cancer. World J Surg 35: 2051-2057, 2011

19. Simon I, Katsaros D, Rigault de la Longrais I, et al: B7-H4 is over-expressed in early-stage ovarian cancer and is independent of CA125 expression. Gynecol Oncol 106: 334-341, 2007.
20. Zang X, Thompson RH, Al-Ahmadie HA, et al: B7-H3 and B7x are highly expressed in human prostate cancer and associated with disease spread and poor outcome. Proc Natl Acad Sci USA 104: 19458-19463, 2007.

21. Galazka K, Oplawski M, Windorbska W, et al: The immunohistochemical analysis of antigens such as RCAS1 and B7H4 in the cervical cancer nest and within the fibroblasts and macrophages infiltrating the cancer microenvironment. Am J Reprod Immunol 68: 85-93, 2012.

22. Awadallah NS, Shroyer KR, Langer DA, et al: Detection of B7-H4 and p53 in pancreatic cancer: potential role as a cytological diagnostic adjunct. Pancreas 36: 200-206, 2008.

23. He C, Qiao H, Jiang H and Sun X: The inhibitory role of B7-H4 in antitumor immunity: association with cancer progression and survival. Clin Dev Immunol 2011: 695834, 2011.

24. Cheng L, Jiang J, Gao R, et al: B7-H4 expression promotes tumorigenesis in ovarian cancer. Int J Gynecol Cancer 19: 1481-1486, 2009.

25. Hofmeyer KA, Scandiuzzi L, Ghosh K, Pirofski LA and Zang X: Tissue-expressed B7x affects the immune response to and outcome of lethal pulmonary infection. J Immunol 189: 3054-3063, 2012.

26. McGrath MM and Najafian N: The role of coinhibitory signaling pathways in transplantation and tolerance. Front Immunol 3: 47, 2012.

27. Wang X, Hao J, Metzger DL, et al: B7-H4 treatment of T cells inhibits ERK, JNK, p38, and AKT activation. PLoS One 7: e28232, 2012. 